\title{
Fever,Weight Loss and Early Satiety due to Gastric Inflammatory Myofibroblastic Tumor; Case Report and Literature Review
}

\author{
Mohammadreza Mohammad Hoseini-Azar ${ }^{1}$, Marjan Mokhtare*2, Ali Zare-Mirzaie ${ }^{3}$, \\ Abbas Gholami ${ }^{4}$, Shahram Agah ${ }^{5}$, Seyed Mohammad Sadegh Ghafoori ${ }^{6}$
}

1. GI Fellow, Colorectal Research center, Rasoul Akram Hospital, Iran University of Medical Sciences, Tehran, Iran

2. Assistant Professor, Colorectal Research center, Rasoul Akram Hospital, Iran University of Medical Sciences, Tehran, Iran

3. Associate Professor,Department of Pathology, Rasoul Akram Hospital, Iran University of Medical Sciences, Tehran, Iran

4. Assistant Professor,Department of Internal Medicine, Rasoul Akram Hospital, Iran University of Medical Sciences, Tehran, Iran

5. Professor, Colorectal Research center, Rasoul Akram Hospital, Iran University of Medical Sciences, Tehran, Iran

6. Medical student, Colorectal Research center, Medical Student Research Committee (MSRC), Faculty of Medicine, Iran University of Medical Sciences, Tehran, Iran

\footnotetext{
* Corresponding Author:

Marjan Mokhtare, MD Assistant Professor, Colorectal Research Center, Rasoul Akram Hospital, Iran University of Medical Sciences, Tehran, Iran Telfax: + 982166554790 Email: marjanmokhtare@yahoo.com Received: 18 Nov. 2015 Accepted: 08 Feb. 2016
}

\section{ABSTRACT}

Gastric inflammatory myofibroblastic tumor (IMT) is a rare tumor with and unpredictable prognosis usually find in young adults. We present an 18-yearold man with gastric IMT. He complained of epigastric pain, intermittent fever and night sweating associated with weight loss since two years ago. Physical examination showed anemic and cachestic features with mild abdominal tenderness in palpitation as well as an abdominal mass in epigastrium. Abdominal CT scan revealed a huge mass that was arising from the stomach. Upper endoscopy revealed a submucosal lesion in fundus of stomach of approximately $8 \mathrm{~cm}$. Endoscopic ultrasound showed a large sub-mucosal non homogenous fundal mass with areas of calcification. The patient underwent laparoscopic partial gastrectomy. Histopathologyand immunohistochemistryevaluation revealed an IMT.

\section{KEYWORDS}

Stomach, Inflammatory myofibroblastic tumor, Fever, Weight loss

Please cite this paper as:

Mohammadhoseini-Azar MR, Mokhtare M, Zare-Mirzaie A, Gholami A, Agah S, Ghafoori SMS. Fever,Weight Loss and Early Satiety due to Gastric InflammatoryMyofibroblastic Tumor; Case Report and Literature Review. Middle East J Dig Dis 2016;8:138-142. DOI: $10.15171 /$ mejdd.2016.19

\section{INTRODUCTION}

Inflammatory myofibroblastic tumor (IMT) is a rare mesenchymal solid neoplasm with undetermined behavior often manifesting in children and young adults. ${ }^{1,2}$ This condition was described for the first time in 1938 by Bunn. ${ }^{2}$ IMT also named inflammatory myofibroblastoma, inflammatory pseudotumor, plasma cell granuloma, and inflammatory myofibrohistiocytic proliferation, each depicting one aspect of the nature of this lesion. ${ }^{3,4}$ Over time, the prognosis of this condition has altered from a reactive, inflammatory process to a neoplasm with intermediate biologic potential. ${ }^{5}$ Various pathogenetic factors are proposed for this condition such as allergic, immunologic, and infectious pathways. ${ }^{6}$ But the exact etiology still remains uncertain. Its biological behavior is unpredictable with a tendency for invasion to adjacent organs and local recurrence $1-3$ but seldom metastases. ${ }^{5}$

In histopathological examination the lesion is composed of spindle cells, myofibroblasts, plasma cells, lymphocytes, eosinophils, histiocytes ${ }^{2,3}$ and variable amounts of fibrosis, necrosis and granulomatous reaction. ${ }^{7}$ IMT commonly originates from the lung and orbit, but it can also be seen in nearly all parts of the body.Extrapulmonary IMTs are not common and gastric IMT is a very rare tumor entity in adults. ${ }^{2,8}$

The presentation of IMT is non-specific and depend on the site of origin. Most cases are accompanied by constitutional symptoms and signs of an in- 
flammatory process such as fever or weight loss and if it originated from the gastrointestinal tract it could present with anemia, GI obstruction, positive fecal occult blood,chest pain, and intussusceptions. Herein, wereport a case of gastric IMT in a young adult with a review of the literature.

\section{CASE REPORT}

An 18-year-old man with a history of nausea, epigastric pain, weight loss, early satiety, weakness, intermittent fever, and night sweating since two years ago was referred to the gastrointestinal ward of our hospital. On physical examination he looked pale and cachectic, with body mass index (BMI) of $18 \mathrm{~kg} / \mathrm{m}^{2}$. During abdominal examination a palpable abdominal mass was found in his epigastrium, and was otherwise normal. His medical history was unremarkable except for an episode of hematemesis six months ago. Laboratory studies revealed microcytic anemia with hemoglobin of $7 \mathrm{mg} / \mathrm{dl}$ on admission and other laboratory results were unremarkable. The chest radiograph was normal.

He underwent esophagogastroduodenoscopy, revealing a large $(8-10 \mathrm{~cm})$ sub-mucosal mass with a fragile surface at the fundus with extension to the greater curvature (figure 1). Endoscopic ultrasound (EUS) showed a large sub-mucosal non-homogenous fundal mass with areas of calcificationthat appeared to originate from the muscularispropria (figure 2). An abdominal computed tomography (CT) scan was requested which revealed a large mass in the stomach, without evidence of liver metastases and lymphadenopathy; however, invasion to splenic hilum was reported (figure 3). A diagnosis of submucosal tumor was made prior to surgery. The patient underwent laparoscopic surgical removal of the mass, partial gastrectomy, and splenectomy (figure 4).

Macroscopic examination of the resected portion of the stomach showed partial gastrectomy as well as splenectomy specimens, the former one revealed a submucosal cream colored mass extending to serosa, $10 \mathrm{~cm}$ in the greatest diameter. Hilum of the spleen was also grossly infiltrated by the tumor. No lymph node was included in the specimen. Histopathological evaluation demonstrated eroded gastric mucosa which was underlined by a fascicular spindle cell growth with elongated vesicular nuclei, lacking obvious atypia, and eosinophiliccyto-

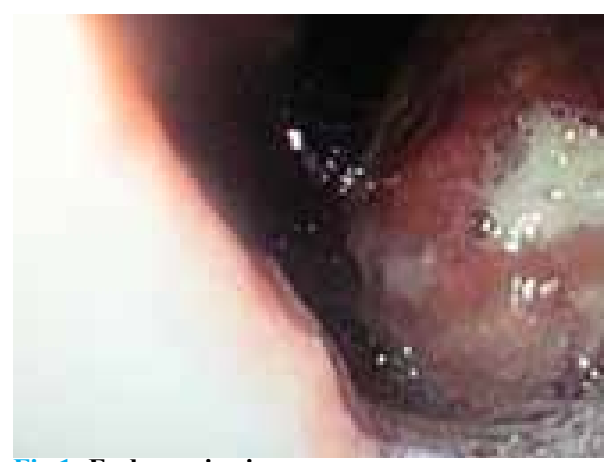

Fig.1: Endoscopic view

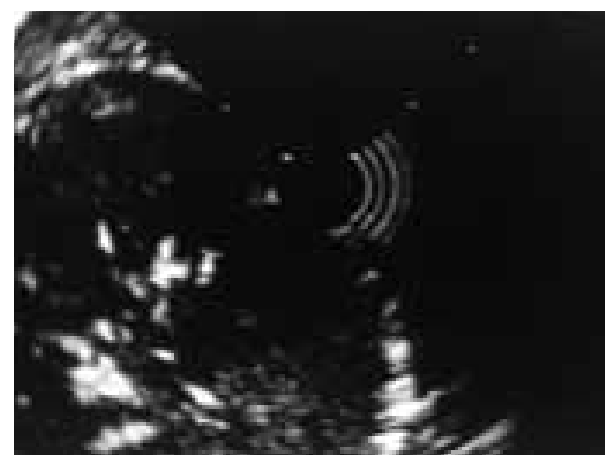

Fig.2: Endosonographic view

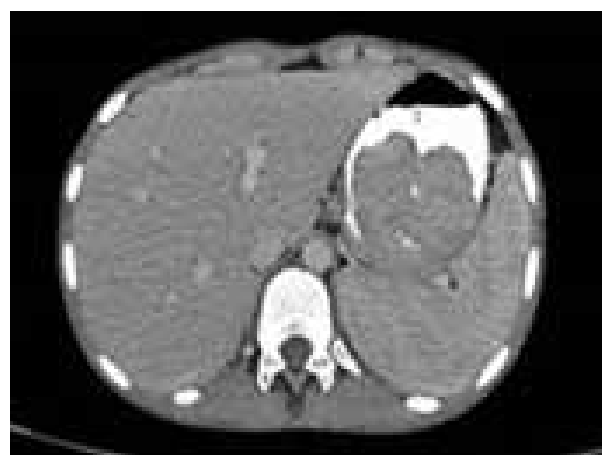

Fig.3: CT scan view

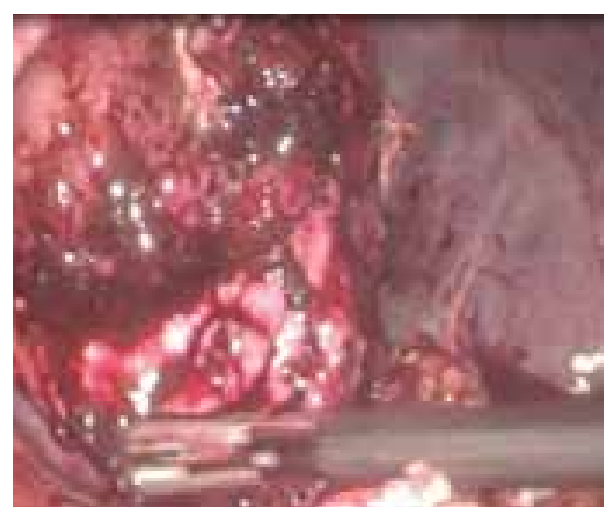

Fig.4: Laparoscopic view 


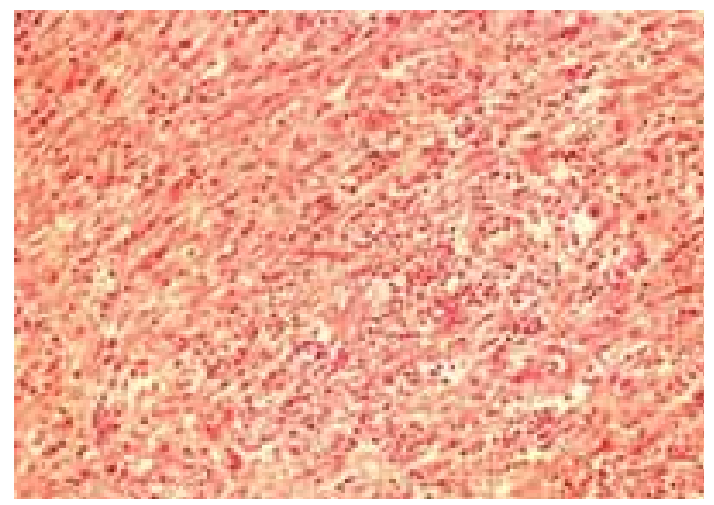

a

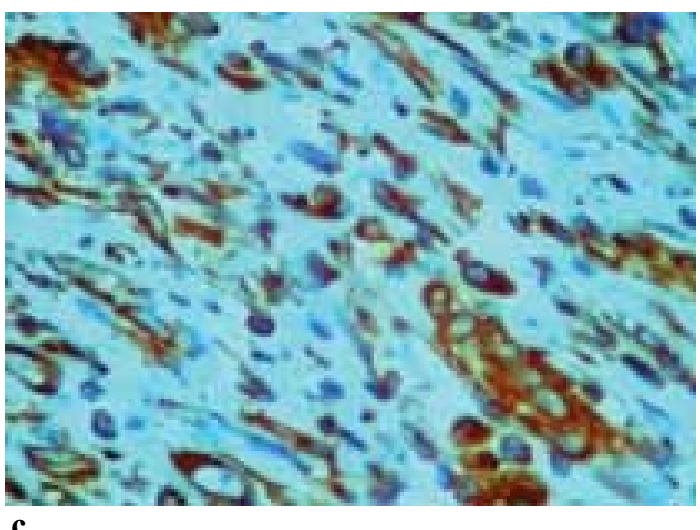

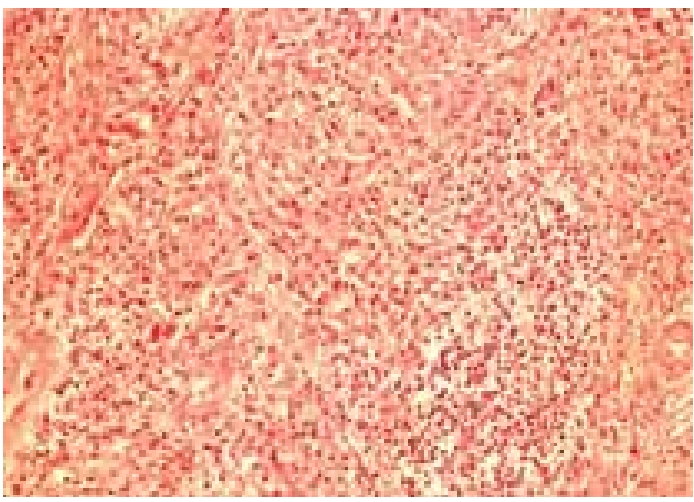

b

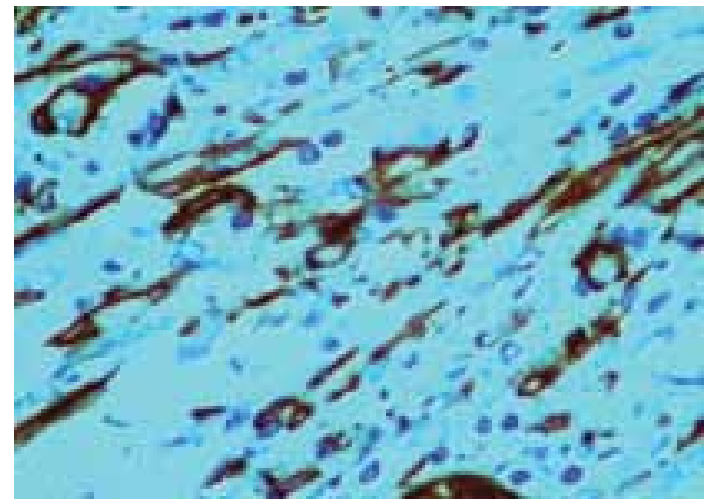

d

Fig.5: Proliferation of spindle cells with elongated vesicular nuclei and eosinophiliccytoplasms were associated with obvious lymphoplasmocytic infiltration (a) with occasional aggregate forms (b).Positive reaction of spindle cells for vimentin (c) and smooth muscle actin (SMA) (d)

plasms associated with abundant lymphoplasmacytic infiltration with occasional aggregate forms(figures $5 \mathrm{a}$ and b).Mitotic figures were scanty (1-2/10 hpfs). Tumor involvedsubmucosa, muscularispropria and serosa of stomach with evidences of splenic parenchymal involvement. Immunohistochemistry revealed positive reaction of tumor cells for vimentin (figure $5 \mathrm{c}$ ), smooth muscle actin (SMA) (figure 5d) and Ki67 (1-2\%) whereas no reaction for CD117, CD34,desmin, anaplastic lymphoma kinase (ALK) protein and GIST-1 (DOG1) was detected. Overall, the histomorphological and immunohistochemical findings were consistent with IMT.

Early postoperative course was uneventful and the patient was discharged from the hospital 9 days after surgery without any surgical related complications. No adjuvant therapy was prescribed. During the next 9 months of follow-up his general condition had improved without any recurrence.

\section{DISCUSSION}

IMT is a rare neoplastic tumor in adults consisting of a variable combination of inflammatory and mesenchymal cells. ${ }^{2,6}$ The main etiology of this condition has not been clarified . This lesions can involve all organs in the body although the gastric form is very rare. ${ }^{5,9}$ Previously it was accepted that IMT is a disease of children and young adults but recent reports indicated that it can occur in all ages. ${ }^{6}$ Almost all gastric IMTs have been detected in patients aged less than 45 years of age. Pulmonary IMT is more common. Nevertheless, it is estimated that the frequency of extra-pulmonary type of this lesion is less than $0.5 \%$ among the general population and abdominal involvement is a more common site. ${ }^{1,6}$ In this context, gastrointestinal region and liver are the common sites of intra-abdominal involvement. ${ }^{5,10}$ Regarding gastric IMT, the tumor can be found in different parts of the stomach. It could invade adjacent organs via the gastric 
wall,as seen in our case. Meanwhile, it had previously been found that womenwere more prone but it has not been confirmed by recent reports. Furthermore, no geographic or ethnic predisposition has been identified.

Clinically, IMT would occur after triggering events such as surgery or trauma, or sometimes it appears in association with other malignancies and systemic inflammatory disorders. ${ }^{2,11}$ In this setting, gastric IMT presents with an abdominal mass with related symptoms, such as abdominal pain, anemia and vomiting depending on the site of involvement and the size of tumour.,12-14 In our case, he presented with abdominal pain along with anemia and weight loss without any predisposing factor. Approximately half of the patients are asymptomatic.

Despite the diagnostic workup, including CT, US, EUS, laboratory analyses, upper flexible endoscopy with biopsy, it was difficult to make an accurate preoperative tumour diagnosis. IMT mimics malignant tumoursin many cases, so most IMT cases require surgical exploration and complete resection to obtain an accurate microscopic diagnosis. $^{1}$

In pathological aspects, the histological characteristics of gastric IMTs are similar to that of soft tissue IMTs. IMT is characterized by proliferation of myofibroblastic cells, infiltration of lymphoplasmocyts and a myxoid background stoma. ${ }^{1,2,5}$ Moreover, based on previous reports three architectural patterns have been described in IMT: myxoid/vascular, compact spindle cell, and hypocellular fibrous (fibromatosis-like). Usually all of them can be found in a single tumor. The myofibroblastic cells in IMTs are spindled and/or epithelioid. Furthermore, cytogenetic abnormalities, such as rearrangements of the ALK gene on chromosome 2p23, DNA aneuploidy as well as clonal chromosome abnormalities, in the pathogenesis of IMT suggest that we face to a potential neoplastic tumor. We did not find these cytogenic abnormalities in our patient. Moreover, ALK positivity is not specific for IMT. It seems that with the presence of ALK, the myofibroblastic proliferation would subside,and in fact,there is no consensus that positive ALK tumors have a less aggressive clinical course.However, expression of ALK and cell morphology rather than other pathological characteristics correlate with the behaviour of IMT. ${ }^{5,10}$

Based on histological studies, spindle cell patterns are almost positive for vimentin.Furthermore in most cases of IMT the smooth muscle actin, muscle-specific actin and desmincan be detected. Moreover, in some cases, CD68 (KP-1), CD30 (Ki-1), cytokeratin, and p53 are positive. These elements may usually be used for diagnosis of IMT and its prognosis., ${ }^{2,9}$ In general, cellular atypia, ganglion-like cells, necrosis,nucleolar prominence, mitotic activity, expression of p53, and anueploidy could be considered as predictors of tumor aggression.

Regarding the differential diagnosis of IMT, there are few gastric tumors that have to be distinguished from IMT. The most important one is gastrointestinal stromal tumor (GIST), followed by inflammatory fibroid polyp, smooth muscle neoplasm, peripheral nerve sheath tumor, solitary fibrous tumor, and fibromatosis. ${ }^{2,4,15}$

The treatment of IMT must includecomplete surgical resectionto reach a definite diagnosis. Although radiation, chemotherapy as well as steroid or nonsteroidal anti-inflammatory drugs (NSAIDs), COX-II inhibitors, thalidomide and cyclosporin have been considered as alternative therapeutic modalities for the treatment of unresectable primary tumors. It is notable that incomplete resection or remnant lesion would be accompanied with risk of recurrence. The recurrence rate of IMT is above $20 \%$ and resection of recurrent lesions is advised. ${ }^{1,16,17}$

In conclusion, IMT is a neoplastic tumor that can evolve to malignancy. In fact, there are no definitive clinical and laboratories characteristics to predict the behavior, recurrence or metastasis of IMT. Complete surgical resection as well as long-term follow-up are advised.

\section{CONFLICT OF INTEREST}

The authors declare no conflict of interest related to this work.

\section{REFERENCES}

1. Bjelovic M, Micev M, Spica B, Babic T, Gunjic D, Djuric A, et al. Primary inflammatory myofibroblastic tumor of the stomach in an adult woman: a case report and review of the literature. World J Surg Oncol 2013;11:35. doi: 10.1186/1477-7819-11-35.

2. Cianci P, Ambrosi A, Fersini A, Tartaglia N, Lizzi V, Sanguedolce $\mathrm{F}$, et al. Voluminous omental inflammatory myofibroblastic tumor in an elderly man: a case report and literature review. Case Rep Surg 2015;2015:873758. doi: $10.1155 / 2015 / 873758$.

3. Leon CJ, Castillo J, Mebold J, Cortez L, Felmer R. Inflammatory myofibroblastic tumor of the stomach: an 
unusual complication after gastrectomy. Gastrointest Endosc 2006; 63:347-9. doi: 10.1016/j.gie.2005.09.026

4. Park SH, Kim JH, Min BW, Song TJ, Son GS, Kim SJ, et al. Exophytic inflammatory myofibroblastic tumor of the stomach in an adult woman: a rare cause of hemoperitoneum. World J Gastroenterol 2008;14:136-9.

5. Gleason BC, Hornick JL. Inflammatory myofibroblastic tumours: where are we now? J Clin Pathol. 2008;61:42837. doi: $10.1136 /$ jcp. 2007.049387

6. Katakwar A, Gedam BS, Mukewar S, Agasti A.Primary gastric inflammatory myofibroblastic tumor in an adultcase report with brief review. Indian J Surg Oncol 2014;5:66-70. doi: 10.1007/s13193-014-0296-5.

7. Coffin CM, Watterson J, Priest JR, Dehner LP. Extrapulmonary inflammatory myofibroblastic tumor (inflammatory pseudotumor). A clinicopathologic and immunohistochemical study of 84 cases. Am J Surg Pathol 1995; 19:859-72.

8. Zhou X, Luo C, Lv S, Gan M. Inflammatory myofibroblastic tumor of the rectum in a 13-month-old girl: a case report. J Pediatr Surg 2011;46:E1-4. doi: 10.1016/j.jpedsurg.2011.03.011.

9. Coffin CM, Hornick JL, Fletcher CD. Inflammatory myofibroblastic tumor: comparison of clinicopathologic, histologic, and immunohistochemical features including ALK expression in atypical and aggressive cases. Am J Surg Pathol 2007;31:509-20. doi: 10.1097/01. pas.0000213393.57322.c7

10. Cook JR, Dehner LP, Collins MH, Ma Z, Morris SW, Coffin $\mathrm{CM}$,et al. Anaplastic lymphoma kinase (ALK) expression in the inflammatory myofibroblastic tumor: a comparative immunohistochemical study. Am J Surg Pathol 2001;25:1364-71.

11. Kiryu S, Takeuchi K, Shibahara J, Uozaki H, Fukayama $\mathrm{M}$, Tanaka $\mathrm{H}$, et al. Epstein-Barr virus-positive inflammatory pseudotumour and inflammatory pseudotumour-like follicular dendritic cell tumour. Br J Radiol 2009;82:e6771. doi: $10.1259 / \mathrm{bjr} / 66918927$

12. Koirala R, Shakya VC, Agrawal CS, Khaniya S, Pandey SR, Adhikary S, et al. Retroperitoneal inflammatory myofibroblastic tumor. Am J Surg 2010;199:e17-9. doi: 10.1016/j.amjsurg.2009.04.014.

13. Ribeiro MC, Lopes LR, de Souza Neto JC, Meirelles LR, de Carvalho RB, Andreollo NA. Rare gastric inflammatory myofibroblastic tumor in an adult woman: a case report with review of the literature. Case Rep Med 2012;2012: 374070. doi: 10.1155/2012/374070.

14. Cho MY, Min YK, Kim NR, Cho SJ, Kim HK, Lee KC, et al. Fever of unknown origin as a presentation of gastric inflammatory myofibroblastic tumor in a two-year-old boy. J Korean Med Sci 2002;17: 699-703. doi: 10.3346/jkms. 2002.17.5.699

15. Dow N, Giblen G, Sobin LH, Miettinen M. Gastrointestinal stromal tumors: differential diagnosis. Semin Diagn Pathol 2006;23:111-9. doi: 10.1053/j.semdp.2006.09.002
16. Mier Odriozola JM, Cortés Julián G, Zotes Valdivia V, Guzmán de Alba E. [Inflammatory myofibroblastic tumor (IMT) of main carina: complete resection by carinectomy and reconstruction]. Gac Med Mex 2014;150 Suppl 2:171-4.

17. Chaudhary P. Mesenteric inflammatory myofibroblastic tumors. Ann Gastroenterol 2015;28:49-54. 